\title{
An Empirical Approach to the Normality of $\pi$
}

\author{
David H. Bailey, Jonathan M. Borwein, Cristian S. Calude, Michael J. Dinneen, \\ Monica Dumitrescu, and Alex Yee
}

CONTENTS

1. Introduction

2. Normality of Real Numbers

3. The Champernowne Number and Relatives

4. Normality for Strings

5. Testing Normality of Prefixes of $\pi$

6. Normality of $\pi$

7. Conclusion

Acknowledgments

References

2000 AMS Subject Classification: 11K16, 65-05, 68Q30

Keywords: normal real, normal string, pi, Poisson process
Using the results of several extremely large recent computations [Yee and Kondo 11], we tested positively the normality of a prefix of roughly four trillion hexadecimal digits of $\pi$. This result was used by a Poisson process model of normality of $\pi$ : in this model, it is extraordinarily unlikely that $\pi$ is not asymptotically normal base 16 , given the normality of its initial segment.

\section{INTRODUCTION}

The question whether (and why) the digits of well-known constants of mathematics are statistically random in some sense has long fascinated mathematicians. Indeed, one prime motivation in computing and analyzing digits of $\pi$ is to explore the age-old question whether and why these digits appear "random." The first computation on ENIAC in 1949 of $\pi$ to 2037 decimal places was proposed by John von Neumann to shed some light on the distribution of $\pi$ (and of $e$ ) [Berggren et al. 04, pp. 277-281].

Since then, numerous computer-based statistical checks of the digits of $\pi$ have failed to disclose any deviation from reasonable statistical norms. See, for instance, Table 1, which presents the counts of individual hexadecimal digits among the first trillion hex digits, as obtained by Yasumasa Kanada. By contrast, early computations revealed provable abnormalities in the behavior of $e$ [Borwein and Borwein 98, Section 11.2]. Figure 1 shows $\pi$ as a random walk drawn as we describe below.

We use the normality for strings introduced and studied in [Calude 94]: a sequence whose prefixes are normal is normal, but the converse is not true. Using the results of several extremely large recent computations [Yee and Kondo 11], we tested positively the normality of a prefix of roughly four trillion hexadecimal digits of $\pi$. This result was used by a Poisson process model of normality of $\pi$ : in this model, it is extraordinarily unlikely that $\pi$ is not asymptotically normal base 16 , given the normality of its initial segment. 


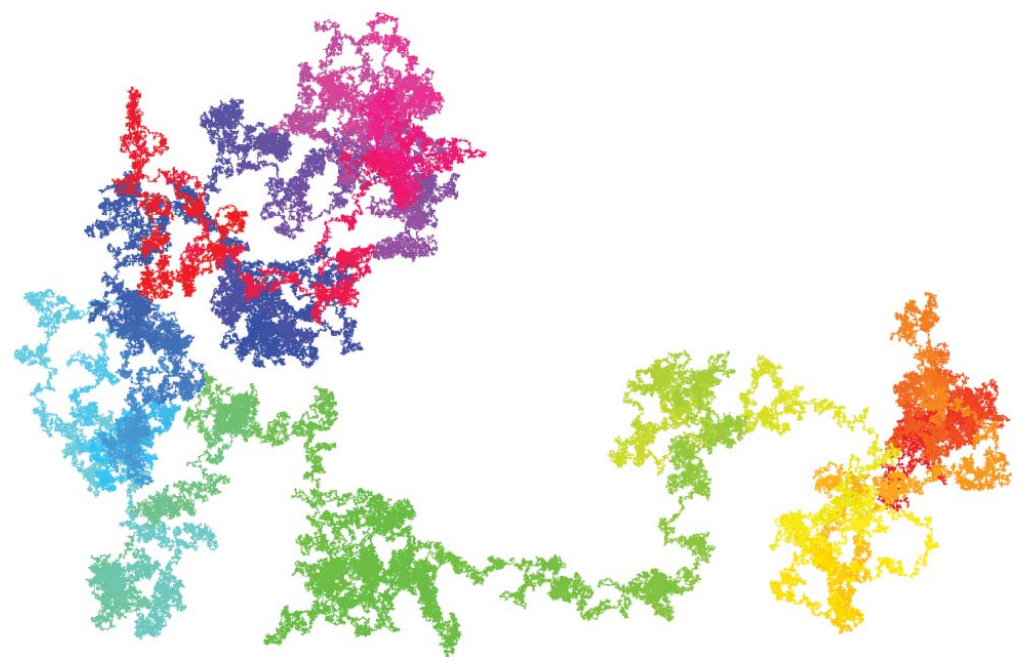

FIGURE 1. A random walk on the first two billion bits of $\pi$ (normal?) (color figure available online).

\section{NORMALITY OF REAL NUMBERS}

In Figures 1 through 4, a digit string for a given number is used to determine the angle of unit steps (multiples of 120 degrees base 3, 90 degrees base four, etc.), while the color is shifted up the spectrum after a fixed number of steps (light and dark, and red-orange-yellow-green-cyanblue-purple-red in the online version). In Figure 1, we

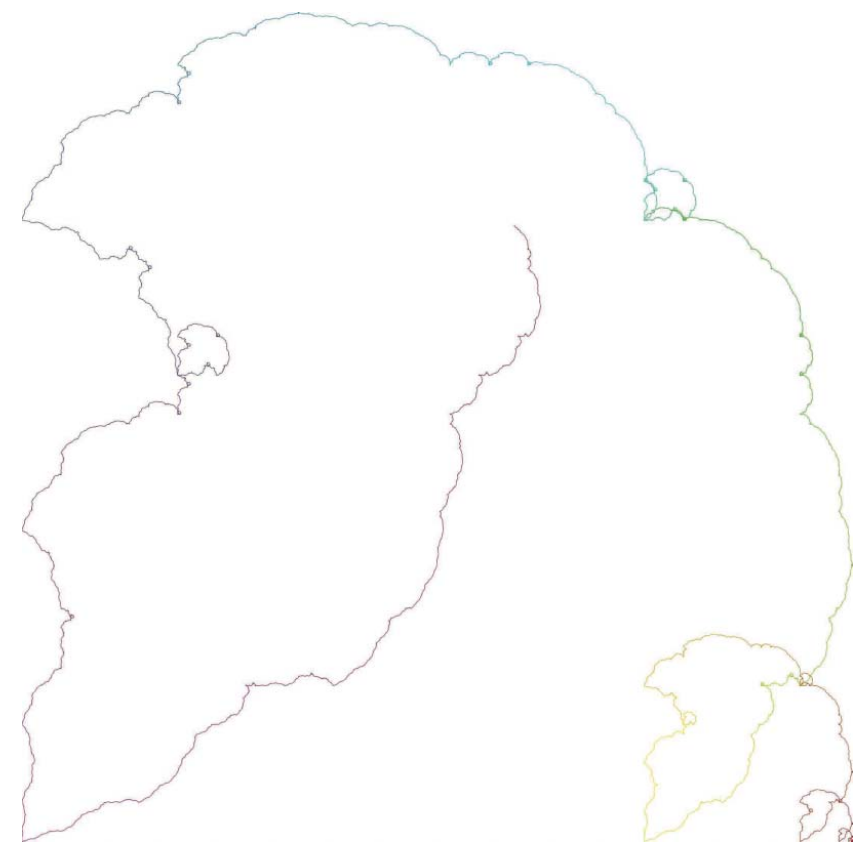

FIGURE 2. A 600000 -step walk on Champernowne's number base 4 (normal) (color figure available online). show a walk on the first billion base- 4 digits of $\pi .{ }^{1}$ We note that the random walks in Figures 2 and 4 look entirely different from the expected behavior of a genuine pseudorandom walk as in Figure 5, which is similar to the random walk in Figure 1.

In the following, given some positive integer base $b$, we will say that a real number $\alpha$ is $b$-normal if every string of base- $b$ digits of length $m$ appears in the base- $b$ expansion of $\alpha$ with precisely the expected limiting frequency $1 / b^{m}$. It follows, from basic measure theory, that almost all real numbers are $b$-normal for any specific base $b$ and even for all bases simultaneously. But proving normality for specific constants of interest in mathematics has proven remarkably difficult.

Borel was the first to conjecture that all irrational algebraic numbers are $b$-normal for every integer $b \geq 2$. Yet not a single instance of this conjecture has ever been proven. We do not even know for certain whether the limiting frequency of zeros in the binary expansion of $\sqrt{2}$ is one-half, although numerous large statistical analyses have failed to show any significant deviation from statistical normals.

Recently, two of the present authors, together with Richard Crandall and Carl Pomerance, proved the following: If a real number $y$ has algebraic degree $D>$ 1 , then the number $\#(|y|, N)$ of 1-bits in the binary

\footnotetext{
${ }^{1}$ This may be viewed in more detail online at http://carma.
} newcastle.edu.au/walks/. 


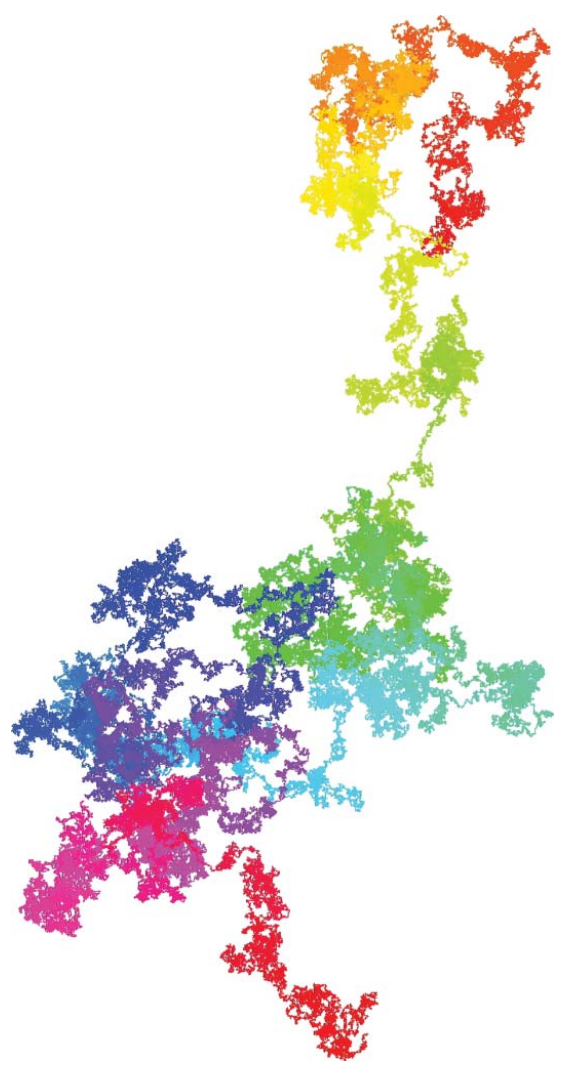

FIGURE 3. A million-step walk on $0.23571113 \ldots$ base 2 (normal?) (color figure available online).

expansion of $|y|$ through bit position $N$ satisfies

$$
\#(|y|, N)>C N^{1 / D}
$$

for a positive number $C$ (depending on $y$ ) and all sufficiently large $N$ [Bailey et al. 04]. For example, there must be at least $\sqrt{N}$ 1-bits in the first $N$ bits in the binary expansion of $\sqrt{2}$, in the limit. A related and more

\begin{tabular}{cr|cr} 
Hex Digits & Occurrences & Hex Digits & Occurrences \\
\hline 0 & 62499881108 & 8 & 62500216752 \\
1 & 62500212206 & 9 & 62500120671 \\
2 & 62499924780 & A & 62500266095 \\
3 & 62500188844 & B & 62499955595 \\
4 & 62499807368 & C & 62500188610 \\
5 & 62500007205 & D & 62499613666 \\
6 & 62499925426 & E & 62499875079 \\
7 & 62499878794 & F & 62499937801 \\
\hline Total: & \multicolumn{3}{|c}{1000000000000}
\end{tabular}

TABLE 1. Digit counts in the first trillion hexadecimal (base16) digits of $\pi$. Note that deviations from the average value 62500000000 occur only after the first six digits, as expected from the central limit theorem. refined result has been obtained by Hajime Kaneko, of Kyoto University, in Japan. He obtained the bound in

$$
\frac{C(\log N)^{3 / 2}}{(\log (6 D))^{1 / 2}(\log \log N)^{1 / 2}}
$$

and extended his results to a very general class of bases and algebraic irrationals [Kaneko 10]. However, each of these results falls far short of establishing $b$-normality for any irrational algebraic number in any base $b$, even in the single-digit sense.

The same can be said for $\pi$ and other basic constants, such as $e, \log 2$, and $\zeta(3)$. Clearly, any result (one way or the other) for one of these constants would be a mathematical development of the first magnitude.

We record the following known stability result [Borwein and Bailey 08, pp. 165-166].

Theorem 2.1. If $\alpha$ is normal in base $b$, and $r, s$ are positive rational numbers, then $r \alpha+s$ is also normal in base $b$.

\section{THE CHAMPERNOWNE NUMBER AND RELATIVES}

The first mathematical constant proven to be 10-normal is the Champernowne number, which is defined as the concatenation of the decimal values of the positive integers, i.e., $C_{10}=0.12345678910111213141516 \ldots$, which can also be written as

$$
C_{10}=\sum_{n=1}^{\infty} \sum_{k=10^{n-1}}^{10^{n}-1} \frac{k}{10^{k n-9 \sum_{\substack{n=0 \\ k=0}} 10^{k}(n-k)}} .
$$

Champernowne proved in 1933 that $C_{10}$ is 10-normal [Champernowne 33]. This was later extended to base- $b$ normality (for base- $b$ versions of the Champernowne constant).

In 1946, Copeland and Erdős established that the corresponding concatenation of primes $0.23571113171923 \ldots$ and also the concatenation of composites $0.46891012141516 \ldots$, among others, are 10-normal [Copeland and Erdös 46]. In general, they proved the following theorem.

Theorem 3.1. [Copeland and Erdős 46] If $a_{1}, a_{2}, \ldots$ is an increasing sequence of integers such that for every $\theta<1$, the number of $a_{i}$ 's up to $N$ exceeds $N^{\theta}$, for $N$ sufficiently large, then the infinite decimal

$$
0 . a_{1} a_{2} a_{3} \ldots
$$

is normal with respect to the base $\beta$ in which these integers are expressed. 


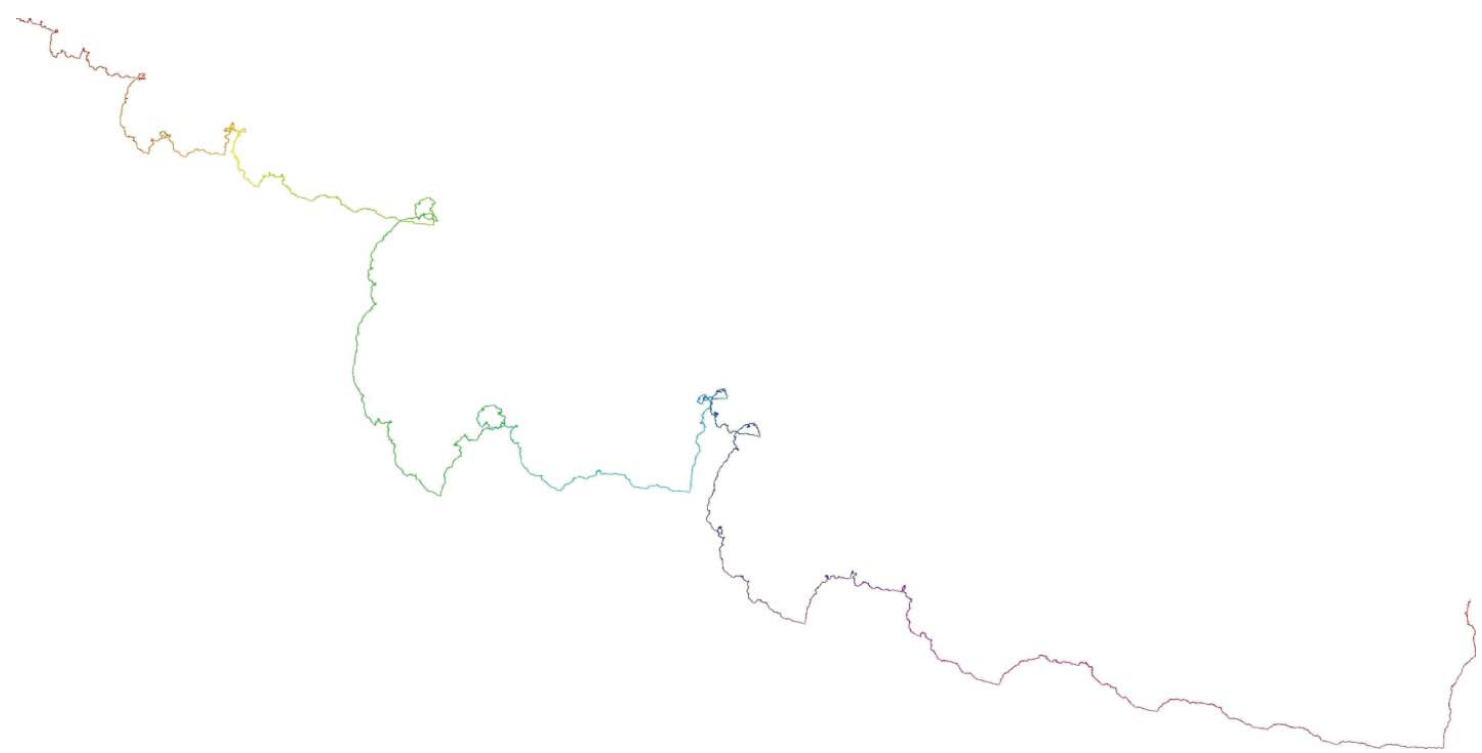

FIGURE 4. A random walk on the first 100000 bits of the primes base two (normal) (color figure available online).

This clearly applies to the Champernowne numbers (Figure 2), to the primes of the form $a k+c$ with $a$ and $c$ relatively prime in any given base, and to the integers that are the sum of two squares (since every prime of the form $4 k+1$ is included).

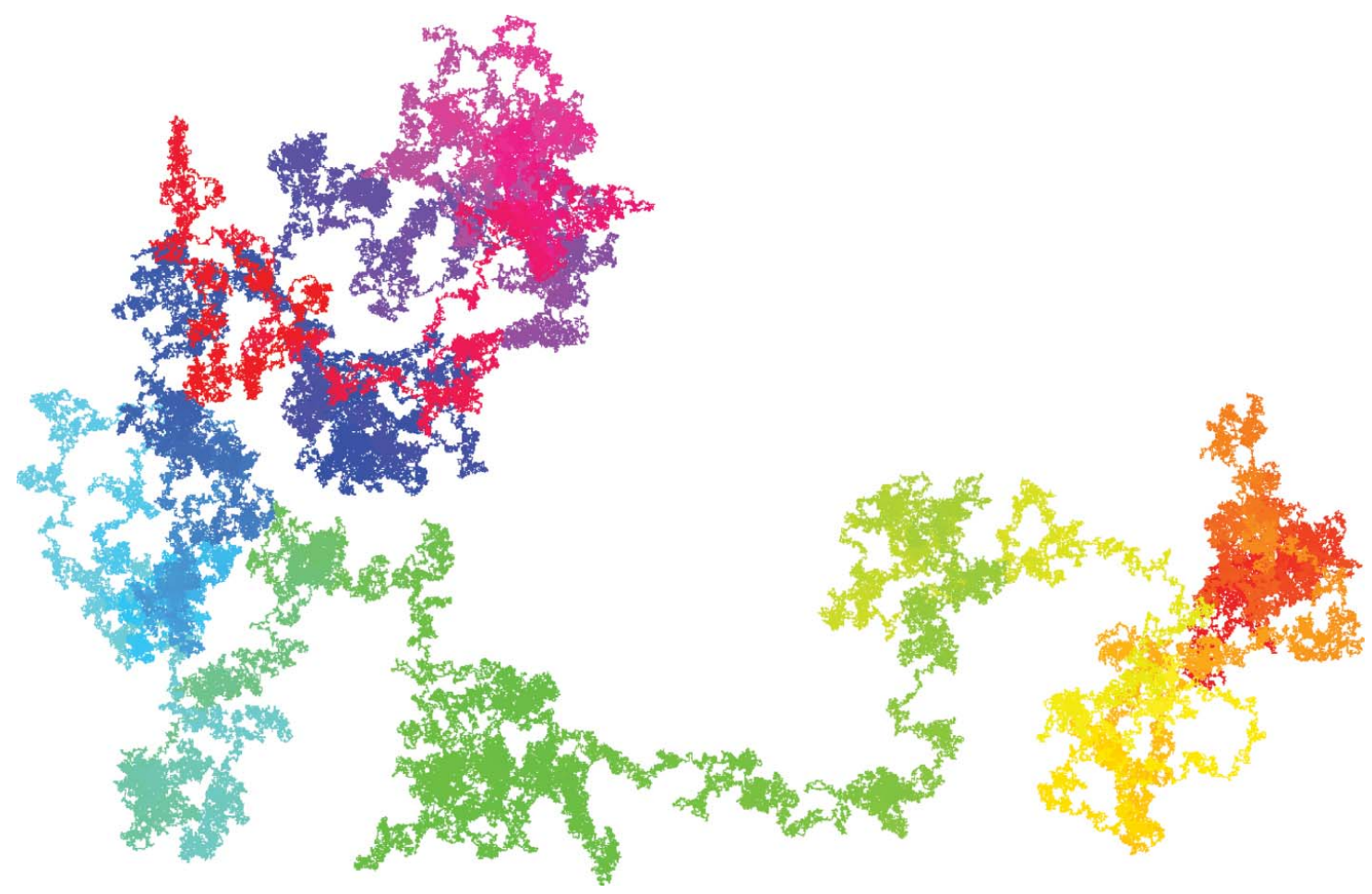

FIGURE 5. A uniform pseudorandom walk (color figure available online). 
Some related results were established by Schmidt, including the following.

Theorem 3.2. [Schmidt 60] Write $p \sim q$ if there are positive integers $r$ and $s$ such that $p^{r}=q^{s}$. If $p \sim q$, then any real number that is p-normal is also q-normal. However, if $p \neq q$, then there are uncountably many $p$-normal real numbers that are not q-normal.

The above result is described in the recent survey [Queffelec 06], which also presents the following theorem.

Theorem 3.3. (Korobov.) Numbers of the form $\sum_{k} p^{-2^{k}} q^{-p^{2^{k}}}$, where $p>1$ and $q>1$ are relatively prime, are $q$-normal.

We are still completely in the dark as to the $b$ normality of "natural" constants of mathematics.

\section{NORMALITY FOR STRINGS}

Let $x$ be a (finite) binary string. We denote by $N_{i}^{m}(x)$ the number of occurrences of the $i$ th string of length $m\left(1 \leq i \leq 2^{m}\right)$, ordered lexicographically, where $|x|_{m}=$ $\lfloor|x| / m\rfloor$ is the number of (contiguous, nonoverlapping) strings in $x$ of length $m$. The prefix of length $n$ of the infinite (binary) sequence $\mathbf{x}=x_{1} x_{2} \ldots x_{m} \ldots$ is denoted by $\mathbf{x} \uparrow n=x_{1} x_{2} \ldots x_{n}$.

Definition 4.1. [Calude 94, Calude 02] Let $\varepsilon>0$ and let $m$ be a positive integer. We say that

1. $x$ is $(\varepsilon, m)$-normal if for every $1 \leq i \leq 2^{m}$,

$$
\left|\frac{N_{i}^{m}(x)}{|x|_{m}}-\frac{1}{2^{m}}\right| \leq \varepsilon
$$

2. $x$ is $m$-normal if for every $1 \leq i \leq 2^{m}$,

$$
\left|\frac{N_{i}^{m}(x)}{|x|_{m}}-\frac{1}{2^{m}}\right| \leq \sqrt{\frac{\log _{2}|x|}{|x|}}
$$

3. $x$ is normal if it is $m$-normal for every $1 \leq m \leq$ $\log _{2}\left(\log _{2}|x|\right)$.

If for every positive integer $n$, the string $\mathbf{x} \uparrow n$ is normal, then $\mathbf{x}$ is normal, but the converse is not necessarily true (because $\mathbf{x}$ can be normal but with a different "speed").

\section{TESTING NORMALITY OF PREFIXES OF $\pi$}

In 1996, one of the present authors (Bailey), together with Peter Borwein (brother of Jonathan Borwein) and Simon Plouffe, published what is now known as the BBP formula for $\pi$ [Bailey et al. 97], [Borwein and Bailey 08, Chapter 3]:

$$
\pi=\sum_{k=0}^{\infty} \frac{1}{16^{k}}\left(\frac{4}{8 k+1}-\frac{2}{8 k+4}-\frac{1}{8 k+5}-\frac{1}{8 k+6}\right) .
$$

We had access to an extremely large dataset, thanks to recent record computations, by Kondo and Yee, of $\pi$ initially to five trillion hexadecimal (base-16) places in August 2010 and then to ten trillion in October 2011 [Yee and Kondo 11]. We first converted these bitswhich Kondo and Yee had confirmed by a computation with (5-1) - to a true binary string of bits using the Python module binascii.

All input lines contained an even number of characters, so it was easy to convert pairs of hexadecimal digits to bytes:

import sys, binascii

for line in sys.stdin.readlines():

sys.stdout.write(binascii.unhexlify(line.strip()))

For our normality test we needed to split a big binary string of length $n$ into $\lfloor n / k\rfloor$ pieces (nonoverlapping strings) of length $k=1,2, \ldots, \log \log n$. We use the term string to denote a binary string of length $k$. We then proceeded to calculate the minimum and maximum frequencies of occurrences of such strings.

This calculation is done by running Algorithm 5.1 once for each different value of $k$.

It is essential to do an efficient streaming implementation of Algorithm 5.1 so that the actual bits of input $X$ are read into main memory only as needed.

Algorithm 5.1: Frequency range of strings of a given length.

Input: Binary string $X$, string length $k$

Output: Minimum and maximum counts over all possible $2^{k}$ strings of length $k$ in string $X$

integer array counts $\left[0, \ldots, 2^{k}-1\right]=[0,0, \ldots, 0]$;

for $i=0$ to $|X|-k$ step $k$ do

$w=$ integer $(X[i, \ldots, i+k-1]) ;$
increment counts $[w] ;$

return $\min ($ counts $), \max ($ counts $)$; 


\begin{tabular}{|c|c|c|c|}
\hline$m$ & Minimum Frequency & Maximum Frequency & Expected Range \\
\hline 1 & $\mathbf{7 9 6 2 9 3 3 1 4 9 1 8 4}$ & $\mathbf{7 9 6 2 9 3 5 3 9 2 2 1 6}$ & $7962907842460, \ldots, 7962960698940$ \\
\hline 2 & 1990732495242 & 1990735357049 & $1990720353555, \ldots, 1990746781795$ \\
\hline 3 & 663576589836 & $\mathbf{6 6 3 5 7 9 0 5 0 1 7 2}$ & $663569046478, \ldots, 663586665305$ \\
\hline 4 & $\mathbf{2 4 8} 841171873$ & $\mathbf{2 4 8} 842651924$ & $248835088899, \ldots, 248848303020$ \\
\hline 5 & $\mathbf{9 9 5 3 5 9 8 9 6 1 1}$ & 99537473460 & $99531392735, \ldots, 99541964032$ \\
\hline
\end{tabular}

TABLE 2. Frequency summary for $N=15925868541400$ bits of $\pi$.

Finally, to check that these minimum and maximum frequencies satisfy the expected range for the normality test, we used the following Python code snippet to generate a table using our earlier formula (4-1):

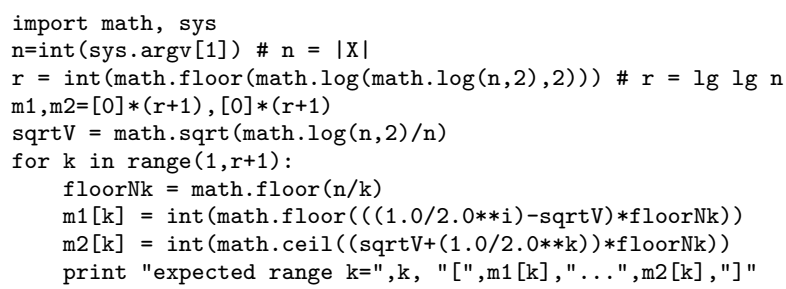

We tested normality for the prefix of $N=$ 15925868541400 bits of $\pi$-nearly 16 trillion bitscalculated with the $y$-cruncher-multi-threaded $p i$ program [Yee 10], and we have found it to be within the normality range as described above. The frequency counts passed our expectedCheck.py test script as shown in Table 2 .

\section{NORMALITY OF $\pi$}

We have tested the prefix of $N=15925868541400$ bits of $\pi$-nearly 16 trillion bits - and we have found it to be normal as described above.

Does this "information" tell us anything about the classical normality of $\pi$ ? In the next subsection, we will use a Poisson process model to provide an affirmative answer to this question.

\subsection{A Poisson Process Model}

We denote by

$$
\mathbf{b}=b(1) b(2) \ldots b(n) \ldots
$$

the (infinite) binary expansion of $\pi$ ( $b$ is a computable function) and by

$$
\mathbf{b} \uparrow n=b(1) b(2) \ldots b(n)
$$

the finite prefix of $\mathbf{b}$ of length $n$.

We base our model on the distribution of 1's and 0's only, i.e., we work with $N_{1}^{1}$ (b「n), the number of occurrences of 1 in $\mathbf{b}\left\lceil n\right.$, so $N_{0}^{1}\left(\mathbf{b}\lceil n)=n-N_{1}^{1}(\mathbf{b}\lceil n)\right.$. A similar, slightly more elaborate, model can be developed for strings of any length.

The number $N_{1}^{1}(\mathbf{b}\lceil n)$ can be connected with $\pi$ by means of a counting (Poisson) process [Ross 83]:

$$
\begin{aligned}
& Y_{n}=\#\{j \mid 1 \leq j \leq n, b(j)=1\}, \quad n=1,2, \ldots, \\
& Y_{0}=0
\end{aligned}
$$

where $Y_{n}=N_{1}^{1}(\mathbf{b} \uparrow n), n=1,2, \ldots$

Theorem 6.1. If $\pi$ is normal, then $\left\{Y_{n}, n=0,1,2, \ldots\right\}$ can be approximated by a homogeneous Poisson process with intensity $\lambda=0.5$.

Proof. By construction, $\left\{Y_{n}, n=0,1,2, \ldots\right\}$ is a Poisson process with an unspecified parameter $\lambda$. Hence $Y_{n}$ is a random variable with parameter $n \lambda$ with the following properties: $E\left(Y_{n}\right)=V\left(Y_{n}\right)=n \lambda, \lim _{n \rightarrow \infty} Y_{n}=\infty$ almost surely.

We apply Chebyshev's inequality, so for every $c>0$,

$$
P\left(\left|Y_{n}-E\left(Y_{n}\right)\right|<c\right) \geq 1-\frac{V\left(Y_{n}\right)}{c^{2}},
$$

we have

$$
P\left(\left|Y_{n}-n \lambda\right|<c\right) \geq 1-\frac{n \lambda}{c^{2}}
$$

Hence

$$
P\left(\left|\frac{Y_{n}}{n}-\lambda\right|<\frac{c}{n}\right) \geq 1-\frac{n \lambda}{c^{2}} .
$$

In view of (4-1), we take

$$
\frac{c}{n}=\varepsilon=\sqrt{\frac{\log _{2} n}{n}},
$$

so we obtain

$$
P\left(\left|\frac{Y_{n}}{n}-\lambda\right|<\varepsilon\right) \geq 1-\frac{n \lambda}{(n \varepsilon)^{2}}=1-\frac{\lambda}{\log _{2} n} .
$$

If $\pi$ is normal, then

$$
\left|\frac{N_{1}^{1}\left(x_{(n)}\right)}{n}-\frac{1}{2}\right| \leq \varepsilon=\sqrt{\frac{\log _{2} n}{n}},
$$


or

$$
\left|\frac{Y_{n}}{n}-\frac{1}{2}\right| \leq \varepsilon=\sqrt{\frac{\log _{2} n}{n}} .
$$

If we identify the random event in relation $(6-1)$ and the certain event in relation $(6-2)$, we get $\lambda=1 / 2$ and

$$
P\left(\left|\frac{Y_{n}}{n}-\frac{1}{2}\right|<\sqrt{\frac{\log _{2} n}{n}}\right) \geq 1-\frac{1}{2 \log _{2} n} .
$$

A Poisson process with intensity $\lambda$ has the following properties [Stoneham 73]:

- The Poisson process $\left\{Y_{n}, n=0,1,2, \ldots\right\}$ has independent increments.

- For $n>r, Y_{n}-Y_{r}$ has a Poisson distribution with parameter $\lambda(n-r)$, and $Y_{n}-Y_{r}$ is independent of $\left\{Y_{t}, t \leq r\right\}$.

Let us denote the positions at which 1's occur (jump moments) by

$$
\tau_{r}=\inf \left\{n \mid Y_{n}=r\right\}, \quad r=1,2, \ldots .
$$

Then

$$
Y_{n}= \begin{cases}0, & \text { if } n<\tau_{1} \\ r, & \text { if } \tau_{r} \leq n<\tau_{r+1}\end{cases}
$$

With the convention $\tau_{0}=0$, we can introduce the $s o$ journ times, or interarrival times,

$$
T_{r}=\tau_{r}-\tau_{r-1}, \quad r=1,2, \ldots .
$$

Note that the sojourn times represent the distances between two successive 1's. Thus, for the string $10^{s} 1$, the sojourn time is $s+1$. Furthermore, the sequence $\left\{T_{r}, r=1,2, \ldots\right\}$ consists of independent identically distributed random variables, with the exponential distribution $\operatorname{Expo}(\lambda)$. Then

$$
E\left(T_{r}\right)=\frac{1}{\lambda}, \quad V\left(T_{r}\right)=\frac{1}{\lambda^{2}} .
$$

Note that the jump moments $\tau_{r}=T_{1}+\cdots+T_{r}$ have an Erlang distribution with parameters $(r ; \lambda)$. Hence

$$
E\left(T_{r}\right)=\frac{r}{\lambda}, \quad V\left(T_{r}\right)=\frac{r}{\lambda^{2}} .
$$

Corollary 6.2. If $\pi$ is normal, then the sojourn times $\left\{T_{r}, r=1,2, \ldots\right\}$ form a sequence of independent identically distributed random variables with the exponential distribution Expo (1/2). Hence

$$
\begin{aligned}
P & \left(T_{r}>t_{r}, r=1, \ldots, k\right) \\
& =\prod_{r=1}^{k}\left(\exp \left(-\frac{t_{r}}{2}\right)\right)=\exp \left(-\frac{1}{2} \sum_{r=1}^{k} t_{r}\right) .
\end{aligned}
$$

\subsection{Testing the Hypothesis That $\pi$ Is Normal}

We test the hypothesis $H$ : " $\pi$ is normal" against the alternative $H_{A}$ : " $\pi$ is not normal." If $H$ is true, then for every $d$, there exists $K_{d}$ such that the sojourn time exceeds the value $d$ if we wait long enough, up to the rank $\left(K_{d}+1\right)$ :

$$
\begin{aligned}
P & \left(T_{1} \leq d, \ldots, T_{K_{d}} \leq d, T_{K_{d}+1}>d \mid H \text { true }\right) \\
& =\prod_{r=1}^{K_{d}}\left(1-\exp \left(-\frac{d}{2}\right)\right) \cdot \exp \left(-\frac{d}{2}\right) \\
& =\exp \left(-\frac{d}{2}\right)\left(1-\exp \left(-\frac{d}{2}\right)\right)^{K_{d}}>0 .
\end{aligned}
$$

We can base our decision of accepting/rejecting normality (hypothesis $H$ ) on the following implication: " $\pi$ is a normal sequence" implies "for every $d$, there exists $K_{d}$ such that $P\left(T_{1} \leq d, \ldots, T_{K_{d}} \leq d, T_{K_{d}+1}>d\right)>0$."

Since we cannot explore the whole sequence $\pi$, we deal with an evidence body represented by a prefix of $\pi$, of length $N$. In this evidence body, we look for the largest value $d_{\max }$ for which a rank $K_{d \text { max }}$ can be identified, or equivalently, we look for the first value $(d+1)$ that is not reached by the sojourn time $T$. Accordingly, the decision of accepting/rejecting the hypothesis $H$ : " $\pi$ is normal" is taken according to the following algorithm:

1. If there is no such $d_{\max }$ in the evidence body, we conclude that the sequence $\pi$ is normal.

2. If $d_{\max }$ and the corresponding $K_{d_{\max }}$ exist, we can decide that the sequence $\pi$ is not normal. The decision is based on the event

$\left\{T_{1} \leq d_{\max }, \ldots, T_{K_{d_{\max }}} \leq d_{\max }, T_{K_{d_{\max }}+1}>d_{\max }\right\}$,

whose probability is

$$
\begin{aligned}
P & \left(T_{1} \leq d_{\max }, \ldots, T_{K_{d_{\max }}} \leq d_{\max }, T_{K_{d_{\max }}+1}>d_{\max }\right) \\
& =\exp \left(-\frac{d_{\max }}{2}\right)\left(1-\exp \left(-\frac{d_{\max }}{2}\right)\right)^{K_{d_{\max }}} .
\end{aligned}
$$

We interpret the above probability to mean that the decision " $\pi$ is normal" has credibility equal to

$$
1-\exp \left(-\frac{d_{\max }}{2}\right)\left(1-\exp \left(-\frac{d_{\max }}{2}\right)\right)^{K_{d_{\max }}} \text {. }
$$




\begin{tabular}{c|ccccccc}
$d$ & 1 & 2 & 3 & 4 & 5 & 6 & 7 \\
\hline$K_{d}$ & 9 & 1 & 14 & 3 & 46 & 56 & 41 \\
$d$ & 8 & 9 & 10 & 11 & 12 & 13 & 14 \\
$K_{d}$ & 78 & 1276 & 446 & 2090 & 18082 & 8633 & 4175 \\
$d$ & 15 & 16 & 17 & 18 & 19 & 20 & 21 \\
$K_{d}$ & 239183 & 5856 & 56453 & 218007 & 643030 & 363117 & 2787207 \\
$d$ & 22 & 23 & 24 & 25 & 26 & 27 & 28 \\
$K_{d}$ & 13733056 & 1003213 & 21127913 & 100317701 & not found & 85745944 & not found \\
$d$ & 29 & & & & & &
\end{tabular}

TABLE 3. Values of $d$ and $K_{d}$ for 400 million bits of $\pi$.

\subsection{Results}

Suppose first that the evidence body is represented by a prefix of 400 million bits of $\pi$. The $d$ values and their corresponding ranks $K_{d}$ are given in Table 3; $\max K_{d}=100317701$.

The value $d=28$ has the property that for every $K$, the event

$$
\left\{T_{1} \leq 28, \ldots, T_{K} \leq 28, T_{K+1}>28\right\}
$$

has not been identified in the evidence body; so, based on the algorithm in Section 6.2 , the decision " $\pi$ is not normal" has credibility

$$
\begin{aligned}
P & \left(T_{s} \leq 27, s=1, \ldots, 100317701, T_{100317702}>27\right) \\
& =\left(1-\exp \left(-\frac{27}{2}\right)\right)^{100317701} \exp \left(-\frac{27}{2}\right) \\
& =2.5576 \times 10^{-66}
\end{aligned}
$$

Suppose now that the evidence body has increased to the prefix of $\pi$ of $N=15925868541400$ bits. The $d$ values and their corresponding ranks $K_{d}$ are given in Table $4 ; \max K_{d}=9274770297096$.

The value $d=43$ has the property that for every $K$, the event

$$
\left\{T_{1} \leq 43, \ldots, T_{K} \leq 43, T_{K+1}>43\right\}
$$

has not been identified in the evidence body, so based on the algorithm in Section 6.2 , the decision " $\pi$ is not normal" has credibility

$$
\begin{aligned}
P( & T_{s} \leq 42, s=1, \ldots, 9274770297096 \\
& \left.T_{9274770297097}>42\right) \\
= & \left(1-\exp \left(-\frac{42}{2}\right)\right)^{9274770297096} \exp \left(-\frac{42}{2}\right) \\
= & 4.3497 \times 10^{-3064} .
\end{aligned}
$$

\begin{tabular}{c|ccccc}
$d$ & 1 & 2 & 3 & 4 & 3 \\
$K_{d}$ & 9 & 1 & 14 & 9 & 46 \\
\hline$d$ & 6 & 7 & 8 & 1276 & 10 \\
$K_{d}$ & 56 & 41 & 78 & 14 & 446 \\
\hline$d$ & 11 & 12 & 13 & 4175 & 15 \\
$K_{d}$ & 2090 & 18082 & 8633 & 19 & 239183 \\
\hline$d$ & 16 & 17 & 18 & 643030 & 363117 \\
$K_{d}$ & 5856 & 56453 & 218007 & 24 & 25 \\
\hline$d$ & 21 & 22 & 23 & 21127913 & 100317701 \\
$K_{d}$ & 2787207 & 13733056 & 1003213 & 29 & 30 \\
\hline$d$ & 26 & 27 & 28 & 611367301 & 1075713943 \\
$K_{d}$ & 273575848 & 85745944 & 234725219 & 34 & 35 \\
\hline$d$ & 31 & 32 & 33 & 15063287853 & 10887127703 \\
$K_{d}$ & 703644000 & 10621041176 & 27019219636 & 39 & 40 \\
\hline$d$ & 36 & 37 & 38 & 1334087352175 & 792460189481 \\
\hline$K_{d}$ & 48115888750 & 19128531469 & 1218723032299 & 44 & 45 \\
\hline$d$ & 41 & 42 & 43 & not found & not found
\end{tabular}

TABLE 4. Values of $d$ and $K_{d}$ for 15925868541400 bits of $\pi$. 


\section{CONCLUSION}

A prime motivation in computing and analyzing digits of $\pi$ is to explore the age-old question whether and why these digits appear "random." Numerous computerbased statistical checks of the digits of $\pi$ have failed to disclose any deviation from reasonable statistical norms. A new avenue for studying the normality of $\pi$ was explored: we proved that the prefix of 15925868541400 bits of $\pi$ is normal when viewed as a binary string [Calude 94].

This result was used in a Poisson process model to show that the probability that $\pi$ is not normal is extraordinarily small, reinforcing the empirical evidence we have presented for the normality of $\pi$. In future work we intend to look methodically at other numerical constants.

\section{ACKNOWLEDGMENTS}

Thanks are due to Dr. Francisco Aragon for his generous assistance with the pictures of random walks. David. H. Bailey was supported in part by the Director, Office of Computational and Technology Research, Division of Mathematical, Information, and Computational Sciences of the U.S. Department of Energy, under contract number DE-AC02-05CH11231.

\section{REFERENCES}

[Bailey et al. 97] David H. Bailey, Peter B. Borwein, and Simon Plouffe. "On the Rapid Computation of Various Polylogarithmic Constants" Mathematics of Computation 66:218 (1997), 903-913.

[Bailey et al. 04] David H. Bailey, Jonathan M. Borwein, Richard E. Crandall, and Carl Pomerance. "On the Binary Expansions of Algebraic Numbers." Journal of Number Theory Bordeaux 16 (2004), 487-518.

[Berggren et al. 04] L. Berggren, J. M. Borwein, and P. B Borwein. Pi: A Source Book, third edition. Springer, 2004.

[Borwein and Bailey 08] J. M. Borwein and D. H. Bailey. Mathematics by Experiment: Plausible Reasoning in the 21st Century, second edition. AK Peters, 2008.
[Borwein and Borwein 98] J. M. Borwein and P. B. Borwein. $P i$ and the AGM: A Study in Analytic Number Theory and Computational Complexity. Wiley, 1998.

[Calude 94] C. S. Calude. "Borel Normality and Algorithmic Randomness." In Developments in Language Theory, edited by G. Rozenberg and A. Salomaa, pp. 113-129. World Scientific, 1994.

[Calude 02] C. S. Calude. Information and Randomness: An Algorithmic Perspective, 2nd edition. Springer, 2002.

[Champernowne 33] D. G. Champernowne. "The Construction of Decimals Normal in the Scale of Ten." Journal of the London Mathematical Society 8 (1933), 254-260.

[Copeland and Erdős 46] A. H. Copeland and P. Erdős. "Note on Normal Numbers." Bulletin of the American Mathematical Society 52 (1946), 857-860.

[Kaneko 10] Hajime Kaneko. "On Normal Numbers and Powers of Algebraic Numbers." Integers 10 (2010), 31-64.

[Queffelec 06] Martine Queffelec. "Old and New Results on Normality." In Dynamics and Stochastics, Lecture Notes, Monograph Series 48, pp. 225-236. Institute of Mathematical Statistics, 2006

[Ross 83] S. M. Ross. Stochastic Processes. Wiley, 1983.

[Schmidt 60] W. Schmidt. "On Normal Numbers." Pacific Journal of Mathematics 10 (1960), 661-672.

[Snyder and Miller 91] D. L. Snyder and M. I. Miller. Random Point Processes in Time and Space. Springer, 1991.

[Stoneham 73] R. Stoneham. "On Absolute $(j, \varepsilon)$-Normality in the Rational Fractions with Applications to Normal Numbers." Acta Arithmetica 22 (1973), 277-286.

[Yee 10] A. J. Yee. "Y-Cruncher-Multi-threaded Pi Program." Available at http://www.numberworld.org/y-cruncher, 2010 .

[Yee and Kondo 11] A. J. Yee and S. Kondo. "10 Trillion Digits of Pi: A Case Study of Summing Hypergeometric Series to High Precision on Multicore Systems." Preprint, available at http://hdl.handle.net/2142/28348, 2011.

David H. Bailey, Lawrence Berkeley National Laboratory, Berkeley, CA 94720. (dhbailey@lbl.gov)

Jonathan M. Borwein, Centre for Computer Assisted Research Mathematics and Its Applications (CARMA), University of Newcastle, Callaghan, NSW 2308, Australia (jonathan.borwein@newcastle.edu.au)

Cristian S. Calude, Department of Computer Science, University of Auckland, Private Bag 92019, Auckland, New Zealand (cristian@cs.auckland.ac.nz) 
Michael J. Dinneen, Department of Computer Science, University of Auckland, Private Bag 92019, Auckland, New Zealand (mjd@cs.auckland.ac.nz)

Monica Dumitrescu, Faculty of Mathematics and Computer Science, University of Bucharest, Bucharest, Romania (mdumi@fmi.unibuc.ro)

Alex Yee, University of Illinois Urbana-Champaign, Urbana, IL, USA. (a-yee@u.northwestern.edu) 